\title{
METHODOLOGY FOR THE EXTRACTION OF HOMOLOGOUS POINTS FROM A DEM/DSM TO EVALUATE THE RELATIVE POSITIONAL ACCURACY
}

Afonso de Paula dos Santos ${ }^{1}$ - ORCID: 0000-0001-7248-4524

Nilcilene das Graças Medeiros ${ }^{1}$ - ORCID: 0000-0003-0839-3729

Aluir Porfírio Dal Poz ${ }^{2}$ - ORCID: 0000-0002-2534-1229

Gérson Rodrigues dos Santos ${ }^{3}$ - ORCID: 0000-0002-4916-0908

Dalto Domingos Rodrigues ${ }^{1}$ - ORCID: 0000-0002-0325-8926

Paulo César Emiliano ${ }^{3}$ - ORCID: 0000-0002-1314-9002

${ }^{1}$ Universidade Federal de Viçosa - UFV, Departamento Eng. Civil, Setor de Eng. de Agrimensura e Cartográfica, Viçosa - MG, Brasil.

E-mail: afonso.santos@ufv.br; nilcilene.medeiros@ufv.br; dalto@ufv.br

${ }^{2}$ Universidade Estadual Paulista - UNESP, Faculdade de Ciência e Tecnologia, Departamento de Cartográfica, Presidente Prudente - SP, Brasil.

E-mail: aluir.dal-poz@unesp.br

${ }^{3}$ Universidade Federal de Viçosa - UFV, Departamento de Estatística, Viçosa - MG, Brasil.

E-mail: gerson.santos@ufv.br; Paulo.emiliano@ufv.br

Received in 05 ${ }^{\text {th }}$ June 2019

Accepted in $17^{\text {th }}$ April 2020

\begin{abstract}
:
This work presents a new methodology for extracting 3D homologous points from a DEM/DSM for relative evaluation of positional accuracies, from terrain features such as ridgelines and hydrography. Firstly, it extracts 3D intersection points between the linear features and then it performs a selection of these homologous points. A discussion regarding the spatial resolution effect of the DEM/DSM on the proposed methodology is carried out. As case study, the planimetric and altimetric positional accuracies evaluation of SRTM (X-band) and Aster GDEM (version 2) DSMs was conducted using, as reference, a DSM obtained via photogrammetry. The SRTM and Aster GDEM (version 2) DSMs showed similar classifications, that is, Class A for the altimetry and Class B for the planimetry at the 1:100,000 scale, according to the Brazilian technical standard of positional accuracy.
\end{abstract}

Keywords: positional accuracy; DSM; DEM; homologous point extraction; spatial data quality.

How to cite this article: SANTOS, A. P.; MEDERIROS, N. G.; DAL POZ, A. P.; SANTOS, G. R.; RODRIGUES, D. D.; EMILIANO, P. C. Methodology for the extraction of homologous points from a DEM/DSM to evaluate the relative positional accuracy. Bulletin of Geodetic Sciences. 26(2): e2020007, 2020 


\section{Introduction}

The growing demand for all sorts of spatial data has enlivened the world cartographic market. This demand includes not only the availability of $2 \mathrm{D}$ information (i.e., a planimetric location or $\mathrm{XY}$ data) but also a significant rise of 3D information (i.e., 3-dimensional location or XYZ data). This expanding interest has consequently improved the production of digital elevation models (DEMs) and digital surface models (DSMs). We must add that in this work DEM is considered as a model representing terrain's surface, immersed or emerged, without neither vegetal cover nor other features (buildings, manmade structures and so on), whilst DSM represents the elevation on the top surface of vegetation cover as well as other features above bare earth (Li et al., 2005; Maune, 2007).

Considering this, the standardization of cartographic products must be highlighted in terms of production of DEMs and DSMs. The current standardization approach utilizes a Spatial Data Infrastructure (SDI), which must be allied to either a law or a technical standard in order to control data quality. For instance, ISO 19157:2013 considers six components of cartographic quality control: positional accuracy, thematic accuracy, temporal quality, completeness, logical consistency and usability. Among them, positional accuracy is the most widely studied element on literature (Ariza \& Atkinson, 2008; Santos et al., 2016b).

Besides, according to Li et al. (2005), it is possible to assess 3D positional accuracy in cartographic products in, at least, two different ways: (i) by evaluating the planimetric and altimetric positional accuracies separately and; (ii) by using a single measure to simultaneously evaluate both planimetric and altimetric accuracies.

As an example of that, in Brazil, Decree 89817 (1984) constitutes regulative instrument to classify a cartographic product concerning its positional accuracy. In this instrument, the positional accuracy classification follows most existing technical standards when it comes to analyzing planimetry and altimetry separately. The positional accuracy evaluation is based on the comparison between a test product and a reference product. In order to do so, checkpoints are used, which are homologous points in both test and reference products.

A substantial problem when evaluating the positional accuracy considering 3D digital models such as DEMs/ DSMs is the identification of homologous points. Santos et al. (2016a) elucidated the following major difficulty: unlike an evaluation of a cartographic database or an orbital image - both of which containing very identifiable cognitive and homologous features concerning the evaluation object in the real world -, there are typically no characteristic features well defined and easy to identify in the field when it comes to a DEM or DSM (at a small to medium scale, i.e.: scales approximately near $1: 25,000$ to $1: 1,000,000$ ) when represented in a matrix format.

In most cases, the evaluation of DEM/DSM is conducted by collecting validation data in places such as crossroads or corner lots, which are usually well defined in the field. However, recognizing an homologous point in a low- to medium-resolution DEM/DSM shows to be a very challenging task. In this case, the evaluation (Figure 1.a) is conducted disregarding the planimetric component. In other words, it is assumed that the planimetry of the DEM is accurate, with only point evaluation left. Hence, the location ( $X$ and $Y$ ) of the checkpoint (circle) is the same as the corresponding point in the DEM (star); thus, an accuracy evaluation of these DEMs considers only discrepancies in the $Z$ coordinate.

On the other hand, the 3D evaluation presented in Figure 1.b incorporates both planimetry and altimetry on the DEM: it considers the DEM corresponding position ( $\mathrm{X}, \mathrm{Y}$ and $\mathrm{Z}$ ) checkpoint (square in Figure 1). Therefore, a measurement of the positional accuracy is obtained by evaluating both planimetric and altimetric components simultaneously. 


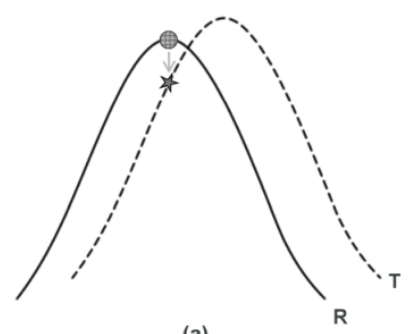

(a)

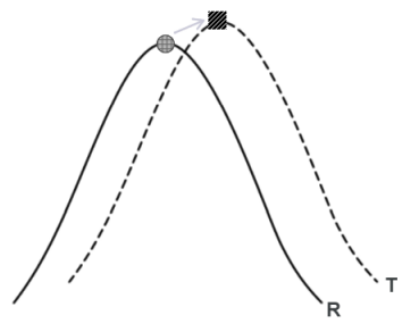

(b)

R-Reference DEM T - Test DEM

Figure 1: Evaluation of positional accuracy of a DEM/DSM. Where (a), the positional accuracy evaluation is carried out only vertically (i.e., not properly); where (b), positional accuracy evaluation is carried out considering planimetry and altimetry.

Among studies regarding DEM/DSM accuracy evaluation, there is loannidis et al. (2014) who evaluated the vertical accuracy of Aster Global Digital Elevation Model version 2 (GDEM2) in Greece using 4,800 checkpoints obtained via GNSS positioning and acquired a root mean square (RMS) of $9.6 \mathrm{~m}$. Moreover, Giacomin et al. (2015) evaluated GDEM2 in Santa Bárbara, Minas Gerais, Brazil, from different interpolating and spatial resolutions while utilizing LiDAR bases as reference; their evaluation average vertical RMS was approximately $24 \mathrm{~m}$. Furthermore, the latter study considered the homogenization step of the geoidal models used in both reference DSM and evaluated DSM.

Rodriguez et al. (2005) obtained a vertical accuracy of $7.5 \mathrm{~m}$ at a $90 \%$ confidence level by comparing the DSM with GPS data in South America. Kolecka and Kozak (2014) evaluated X-band Shuttle Radar Topography Mission (SRTM-X) in a mountainous area of Poland and obtained an average altimetric discrepancy of 9 meters and an RMS discrepancy of 38.4 meters in which $74 \%$ of the tested points presented discrepancies lower than 16 meters (technical specification of SRTM-X). The authors also concluded that the presence of forests may introduce vertical discrepancies of approximately 10 meters. It is important to point out that the abovementioned studies either neglected or failed to explicitly state the planimetric uncertainties in the DSM during the verification of the vertical model accuracy and therefore used an erroneous model, as shown in Figure 1.

Additionally, Tachikawa et al. (2011) evaluated the planimetric and altimetric accuracies on Aster GDEM2 with a more accurate DSM as reference. In order to do so, the authors moved the Aster GDEM over the reference DSM with the lowest elevation discrepancy. The positional difference between original and matched position were treated as planimetric accuracy.

In addition, Dong et al. (2015) evaluated planimetric and altimetric accuracies of SRTM-X and GDEM2 using a more accurate reference DSM for a region in China. The authors registered the SRTM-X and GDEM2 DSMs using the normalized cross-correlation method and, consequently, obtained the average planimetric discrepancy. After that, the altimetry accuracies of the models were evaluated using GPS data.

Those researches mentioned herein evaluated planimetric accuracy regarding the concept of image register without using checkpoints. One of the most efficient approaches for evaluating the quality of a DEM/DSM would be using 3D homologous points; however, due to the difficulty in defining such points, in low- to medium-resolution models, there is negligence of the planimetric quality evaluation.

Based on the above discussion, the aim of this work is to propose a new method to define and extract homologous 3D points used in planimetric and altimetric positional accuracies of a DEM/DSM in raster format. Hence, it specifically proposes: (i) the presentation of a new methodology for the extraction of homologous points in the relative evaluation of a DEM/DSM by utilizing point and linear features and (ii) a study case to evaluate the 3D positional accuracy of an SRTM DSM (X-band) and an Aster GDEM DSM (version 2) using a DSM obtained by photogrammetry as reference. 


\section{3D-checkpoints extraction proposed methodology for DEM/DSM positional assessment}

As mentioned, the majority of altimetric evaluations of a medium-resolution DEM/DSM presuppose the availability of an accurate planimetry, which can cause uncertainties when defining homologous points hence the outcomes may have uncertainties as well.

Santos et al. (2016) mentioned that few studies have evaluated the planimetric accuracies of DEMs/DSMs in raster format at the small and medium scales. Most of the studies evaluating DSM/DEM planimetry use data from LiDAR and are normally carried out in urban areas with large amount of detail.

For large-scaled data, such as a DSM derived from LiDAR, planimetric and altimetric analyses are based on discrepancies in ridgelines from building's roof, as shown by Maas (2002), Vosselman (2008), and Höhle and Pedersen (2010). In addition, Reinoso (2011) and Mozas et al. (2013) have already evaluated the planimetry in DEMs/DSMs based on the analysis of contour lines, which are linear features

Considering uniquely the evaluation of planimetric and altimetric accuracies in low- to medium-resolution DEM/DSM, so far there is no known literature containing methodology for extraction of 3D homologous points. Hence, ridgelines and hydrography -found in a great number of spatial data and presenting well defined geometric shape in most cases-, may be used as homologous features in order to enable a DEM/DSM 3D evaluation.

From these linear features, a high number of points can be extracted characterized by their intersections as ridges and by confluence points in the numerical hydrography. With a set of ridges and confluence points with known XYZ coordinates in test and reference DSMs / DEMs, one can select the homologous points to use as checkpoints for the DEM/DSM assessment.

It is important to point out that Santos et al. (2016) used linear features for evaluating the planimetric positional accuracy in DEM/DSMs. Their recommendation is the usage of points as features to evaluate both planimetric and altimetric accuracies from a DEM/DSM. Despite the usage of point features is considered traditional, the innovation in the work is the suggested approach for defining and extracting of 3D homologous points for the evaluation.

In this sense, Figure 2 shows the flowchart of the methodology proposed in this study for extracting ridges and confluence points. Moreover, Figure 3 illustrates the process to obtain a set of homologous 3D points to be used as checkpoints on the positional accuracy evaluation from a medium-resolution DEM/DSM.

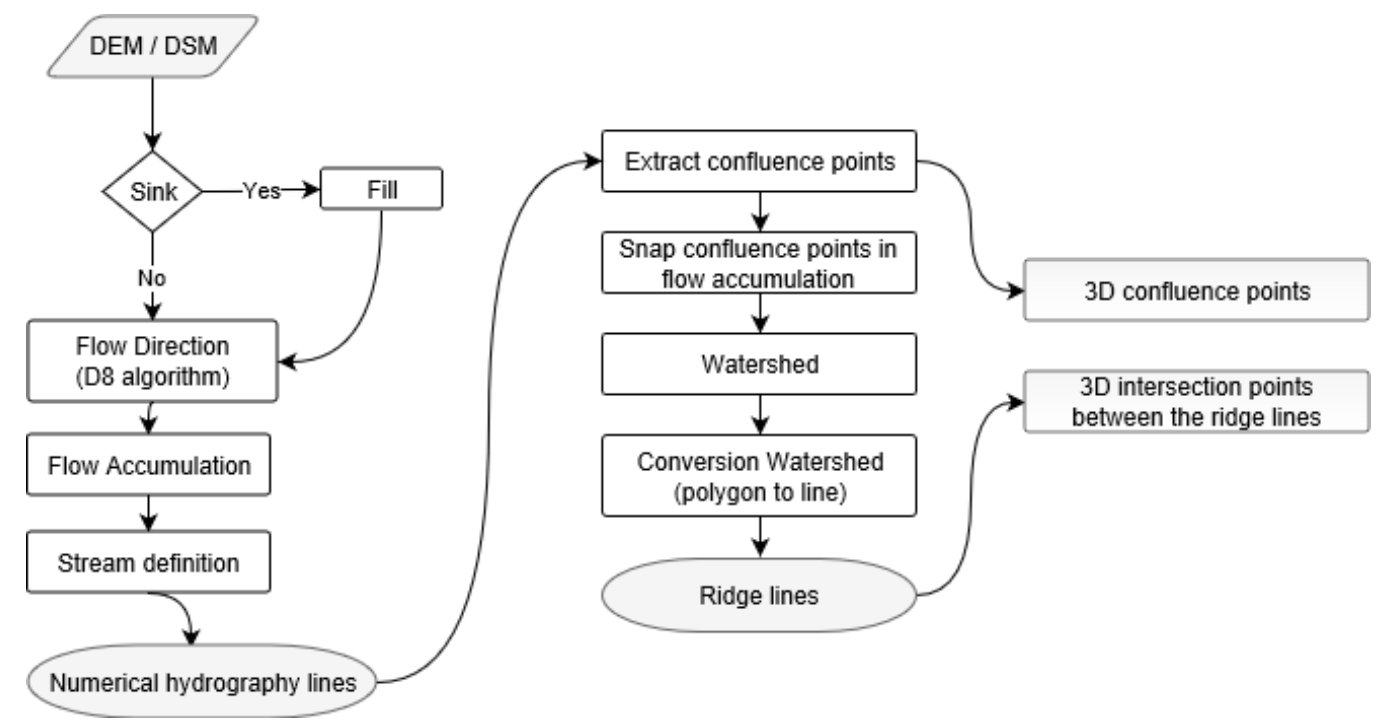

Figure 2: Flowchart for the extraction of three-dimensional ridgeline and confluence points from a DEM/DSM. 
During the process of extracting 3D points in intersection of ridgelines as well as confluence points, the presence of sinks must be verified. If this presence checks out, then one must fill in the sink cell, where its altitude value should match the lowest observed value among the eight adjacent cells. That allows the DSM to be consistent to extract numerical hydrography, which means that all the flow inside an accumulation basin flows to the river mouth.

Subsequently, the flow direction is obtained from the D8 algorithm (O'Callaghan and Mark, 1984) which considers the neighborhood cell with highest slope. Following this, the DSM flow accumulation is computed in a way that each DSM cell is defined by the number of cells upstream that contribute to the flow in a specific position. In order to define a drainage network, a flow accumulation threshold must be defined with the intention to determine the beginning of the watercourse. From the hydrography extracted from the DSM in vector format, one obtains the $3 \mathrm{D}$ confluence points which will be used as possible homologous points during the process of a DSM positional accuracy evaluation.

Following the flowchart presented on Figure 2, there is the extraction of 3D intersection points between ridgelines, which, alongside with the confluence points, are targeted by the proposed methodology. From each confluence point, a 3-cell radius region is analyzed in order to find the cell location containing the highest accumulated flow. This cell will be a confluence point from all the nearby tributaries and it will be used for basin delimitation. Subsequently, the basin polygon is converted into lines representing terrain ridgelines.

Likewise, Figure 3 presents a general flowchart of the method proposed in this paper. A known more accurate DSM must be used as reference so the relative positional accuracy evaluation can be performed. Besides, the process shown in Figure 2 is applied on both reference and test DSM/DEM generating a set of three-dimensional points for both models automatically. With these characteristic 3D terrain points (ridges and confluence points), a manual selection of the homologous points is carried out on both DEMs/DSMs in order to compose a checkpoint sample. This sample is used to evaluate the test DSM positional accuracy.

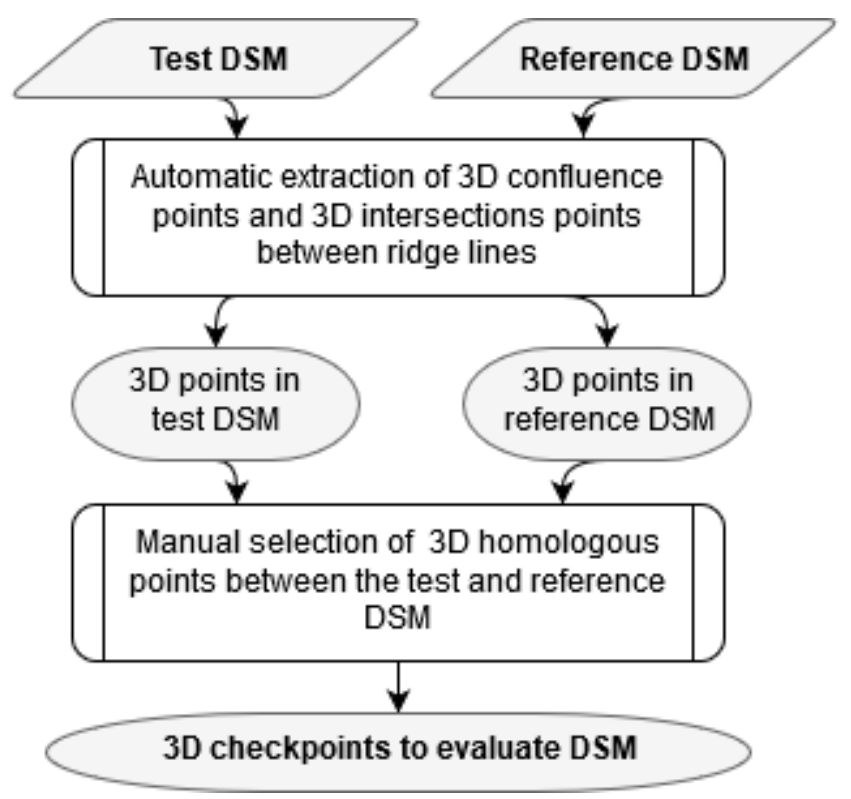

Figure 3: Process to obtain a set of 3D homologous checkpoints to evaluate a DSM.

Additionally, Figure 4 presents a DSM as a representation of the numerical hydrography lines (in blue), ridgelines (in red) and 3D points (in black) extracted from the intersections of ridgelines and the confluences of numerical hydrography lines. Meanwhile, Figure 5 shows the selection of the homologous checkpoints (ridges) extracted from the test and reference DSMs. When visually comparing the correspondence between the ridgelines and the intersection points extracted from both DSMs, it is possible to estimate the planimetric and altimetric 
accuracy levels. If the ridgelines from the reference model are nearby (horizontally) the corresponding test model, the tested DSM will likely have a good planimetric accuracy level. If the ridgelines do not correspond in dimension and shape, the vertical accuracy may be negatively affected.

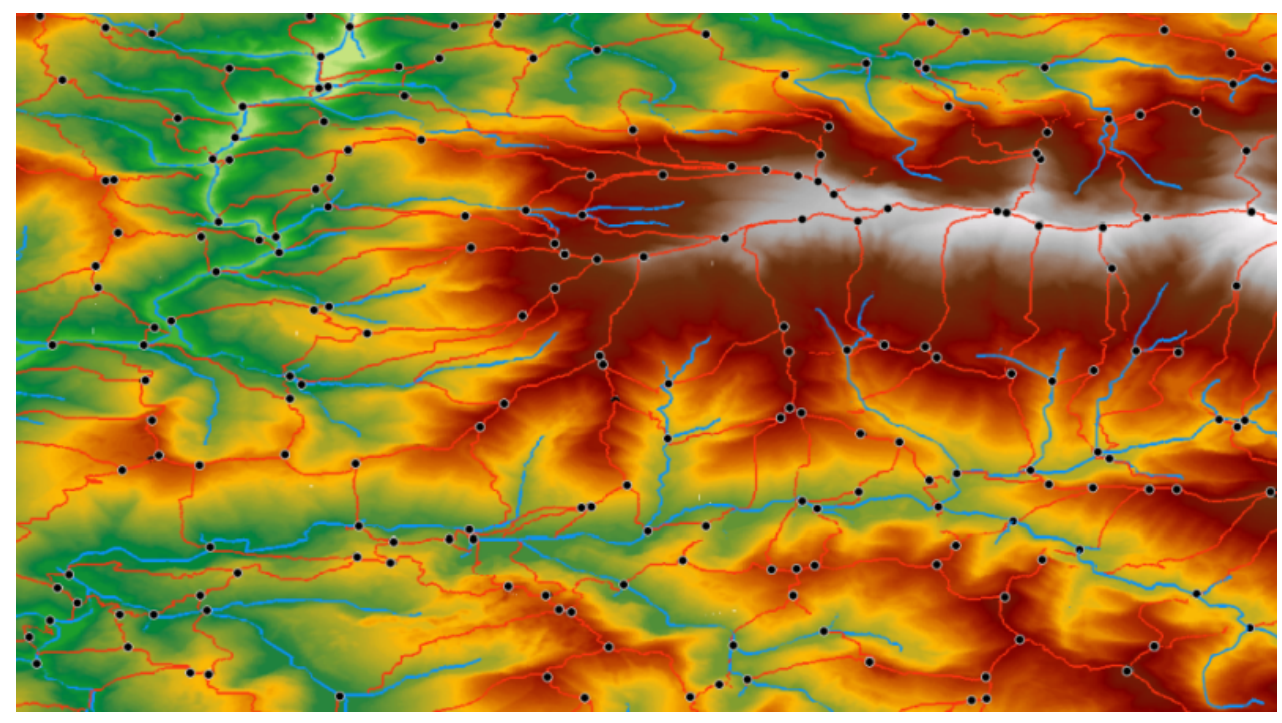

Figure 4: Extracted points (black) of the ridgelines (red) and numerical hydrography lines (blue) from a DSM.

The 3D points extracted from the ridgelines and confluence points selected for an evaluation of the positional accuracy must be spatially distributed horizontally and vertically. Besides, the homologous points must be carefully selected where the ridgelines that define those points are similar in both DSMs in terms of their geometries, dimensions and levels of detail. Another consideration regarding the homologous points is that their selection is based on the altitude of the study area: these points should cover low, medium and high altitudes. Due to the slope levels, the use of ridgelines and numerical hydrography lines is beneficial compared with the analysis approach based on contour lines used by Reinoso (2011) and Mozas et al. (2013).
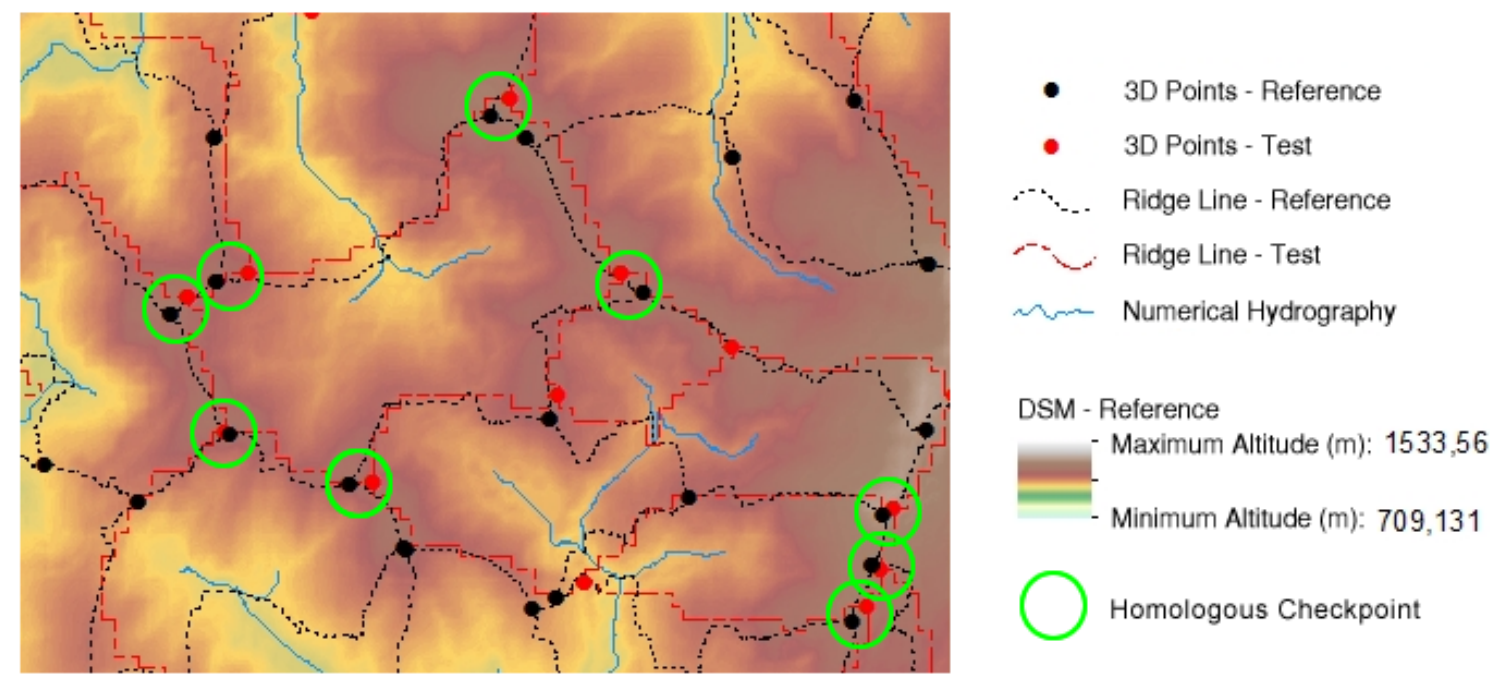

Figure 5: Selection of homologous checkpoints (from ridgelines) for the positional accuracy evaluation.

Typically, the reference DSM has a higher spatial resolution as well as positional accuracy than the test DSM. This can be explained by the fact that the reference DSM is generated by data acquired with higher densities, 
together with greater spatial distribution and precision. Thus, one can question the influence of different resolutions on the proposed methodology.

Murphy et al. (2008), Li and Wong (2010) Boulton and Stokes (2018) and Wilson (2018) point out that higher spatial resolutions in a DEM/DSM lead to a higher accuracies in locations of drainage networks extracted from the model. Likewise, Barber and Shortridge (2005) and Remmel et al. (2008) added that achieving better boundary definitions of river basins and better characterizations of ridgelines is possible with a higher spatial resolution.

Furthermore, Gyasi-Agyei et al. (1995) and Goulden et al. (2014) stated that the DEM/DSM relief becomes generalized (reduced) when its pixel size is increased. As an effect, the feature accuracy extracted from these models is worsened. Examples of such features can be drainage networks, river basis and ridges. Moreover, Goulden et al. (2014) warned that DEM/DSM drainage network will tend to meander unrealistically within the flow channel if the DEM/DSM resolution is below the flow channel width, resulting in deviations in the drainage network location.

In addition to the DEM/DSM resolution, the drainage network is influenced by: (i) the topographical characteristics of the employed relief; (ii) the interpolators; (iii) the method used to obtain the flow direction and (iv) the threshold used to determine the beginning of the flow (Goulden et al., 2014). Hence, in plain reliefs, the use of confluence points extracted from numerical hydrography lines should be evaluated since determining the numerical hydrography may be affected due to the DSM altimetry precision level, under such conditions.

Usually, the D8 algorithm (O'Callaghan and Mark, 1984) is used to obtain the flow direction. However, there are other methods such as the Do algorithm (Tarboton, 1997), shown to be more robust. In this sense, McMaster

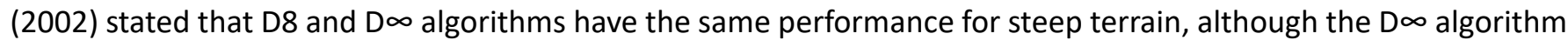
presents better results for flat terrain.

As previously mentioned herein, raster resolution may influence on the extraction of numerical hydrography and ridgelines. Consequently, different resolutions between the test DSM and the reference DSM may influence on the extraction of homologous points. Some assumptions in order to obtain discrepancies between test and reference DSM are: (i) the reference DSM pixel size (PR) is smaller than the test DSM pixel size (PT); (ii) the reference DSM pixel size is a multiple of the test DSM pixel size; (iii) the location of the reference DSM raster structure is compatible with the one of the test DSM; (iv) the altitude (cell value) of the reference DSM is similar to the cell values in the test DSM; (v) the hydrography and ridgeline extraction methods are the same for the reference and test DSM. With these in mind, the planimetric discrepancy $(d)$ can be obtained through the difference between the homologous points $(k)$ of the test $\operatorname{DSM}\left(k_{T}\right)$ and the reference $\operatorname{DSM}\left(k_{R}\right)$ according to Equation 1 :

$$
d_{k_{T}}-d_{k_{R}}=\frac{P T \sqrt{2}}{2\left(\frac{P T}{P R}\right)} \cdot\left(\frac{P T}{P R}-1\right)
$$

When PR tends to zero, the planimetric discrepancy between the homologous points is maximal, and its magnitude is equal to the half-diagonal length of the test DSM pixel size (Equation 2):

$$
d \max _{k_{T}-k_{R}}=\frac{P T \sqrt{2}}{2}
$$

Figure 6 illustrates the planimetric discrepancy (d) that can be caused exclusively by the raster resolution difference. A reference DSM (gray) with a pixel size equals to half the size of the test DSM (red) is shown in Figure 6.a. In Figure 6.b, the ratio between both pixel sizes is equal to 3; in Figure 6.c, this ratio is equal to 5; in Figure 6.d, the ratio is close to zero.

It is important to highlight that this is a hypothetical case. As discussed in the assumptions for Equation 1, all the reference DSM cells overlapping a test DSM cell have the same altitude values, although this will rarely occur. Therefore, the altimetry does not have any influence on this analysis of the positional discrepancy due to the different model resolutions. In a real scenario, the test DSM and reference DSM altitudes will not be equal, and thus, 
estimating the influences of those different resolutions may have values greater or lesser than the one described by Equation 2 depending on the DSM altimetric quality.

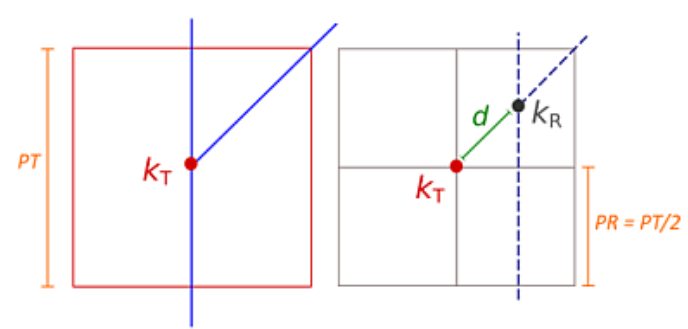

(a)

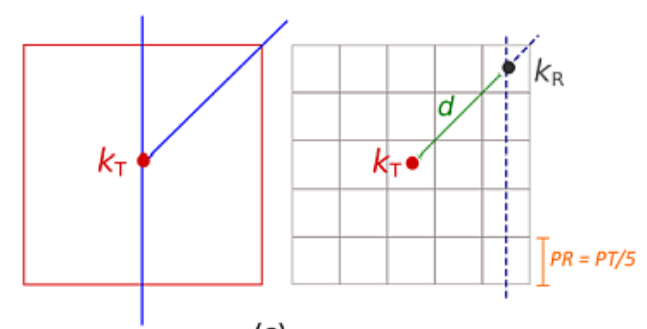

(c)

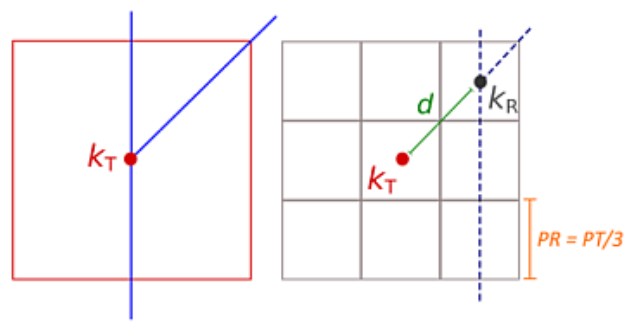

(b)

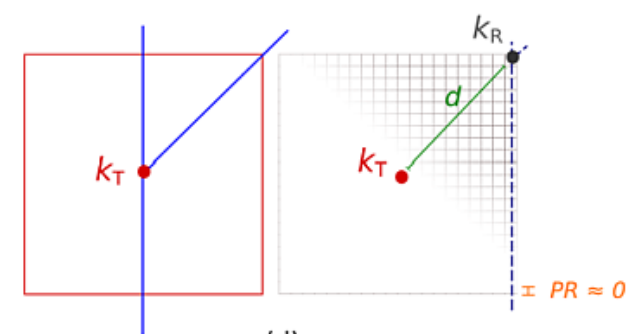

(d)

Figure 6: Planimetric positional discrepancy caused by the resolution difference between the rasters in the extraction of drainage lines and ridgelines.

Another point that should be noted is that the methodology proposed herein for the extraction of homologous points is developed to evaluate the positional accuracy of a low- or medium-resolution DSM, which will produce cartographic information at small scales. According to Kimerling (2011), the relationship between the DEM/DSM resolution and the cartographic scale is linked to the principle of map compilation, where the scale denominator can be obtained by multiplying the DEM/DSM pixel size (in meters) by the pixels density on the map. The abovementioned author suggests this density to be 4,000 pixels per meter. Thus, when a DSM has a $30 \mathrm{~m}$ resolution, the scale will be $1 / 120,000$. Considering that the maximum planimetric discrepancy $(d)$ in Equation 2 ( $21 \mathrm{~m}$ for a $30 \mathrm{~m}$ resolution DSM) is smaller than the graphing error $(0.2 \mathrm{~mm})$ for the scale $(24 \mathrm{~m})$, the proposed method for the extraction of homologous points is not influenced by the difference between the resolutions of the test and reference DSMs.

\section{Materials and Methods}

\subsection{Study area and data used}

The study area encompasses parts of the municipalities of Nova Lima and Rio Acima in the State of Minas Gerais, Brazil (Figure 7). These municipalities contain the Macacos River sub-basin that flows into the São Francisco River hydrographic basin. The study area has $180 \mathrm{~km}^{2}$ with altitudes ranging from 710 up to 1,540 meters, it is delimited by latitudes from $20^{\circ} 00^{\prime} 38^{\prime \prime} \mathrm{S}$ to $20^{\circ} 07^{\prime} 05^{\prime \prime} \mathrm{S}$ and the longitudes from $43^{\circ} 57^{\prime} 28^{\prime \prime} \mathrm{W}$ to $43^{\circ} 48^{\prime} 49^{\prime \prime} \mathrm{W}$, as shown in Figure 7. 

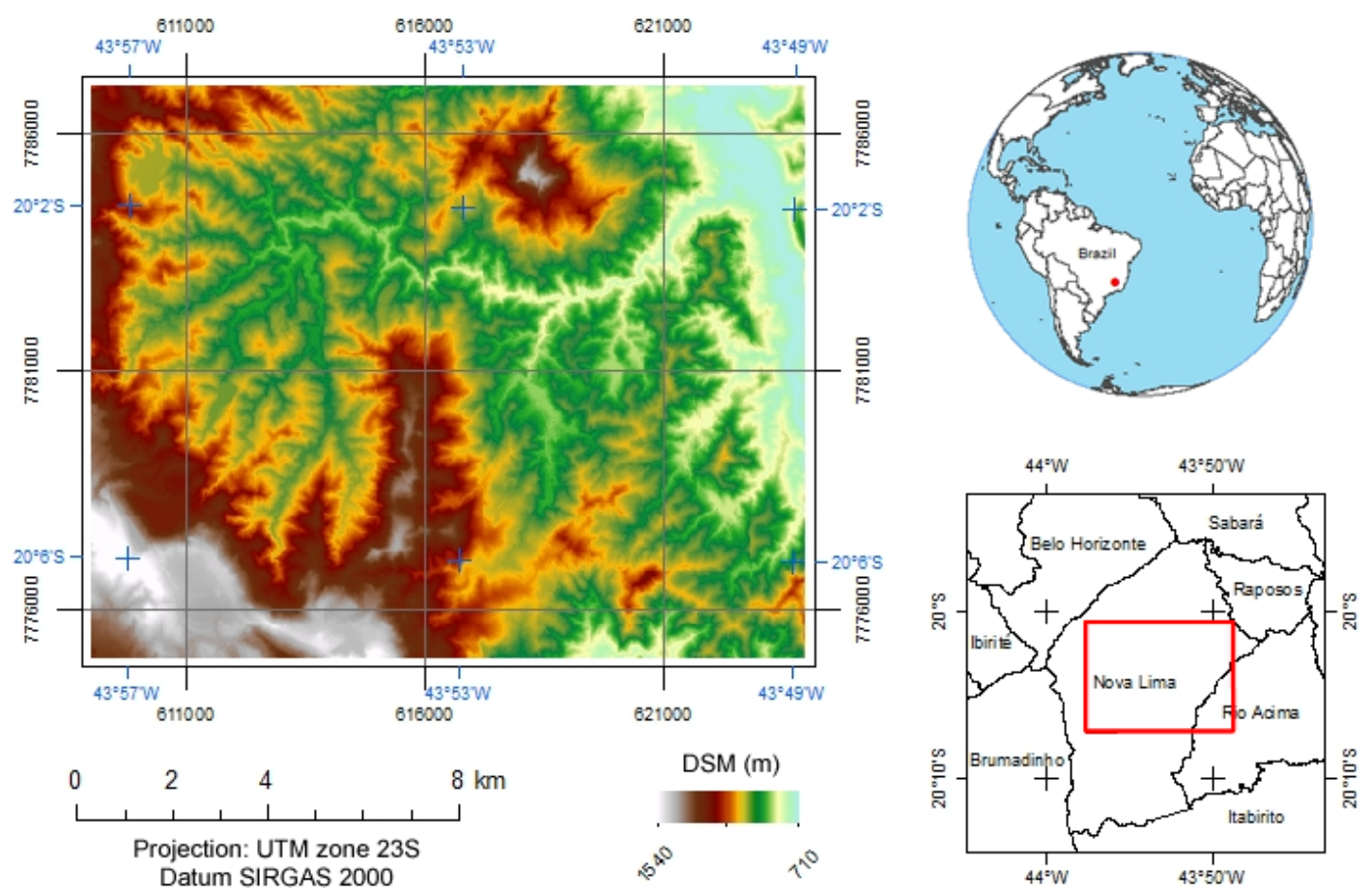

Figure 7: The DSM of the study area, located in the municipalities of Nova Lima and Rio Acima State of Minas Gerais, Brazil.

One of the DSMs evaluated in this work is the X-band Shuttle Radar Topography Mission (SRTM-X) obtained during the month of February, 2000 throughout a spatial mission operated by the National Aeronautics and Space Administration (NASA) in conjunction with the USA Department of Defense represented by the National GeospatialIntelligence Agency (NGA), the German Aerospace Center (DLR) and the Italian Space Agency (ASI). This mission used two types of Synthetic Aperture Radar (SAR) by Interferometry (InSAR); one of the systems contains the X-band (2.4 to $3.8 \mathrm{~cm}$ wavelength) with a spatial resolution of 1 arc second ( 30 meters at the equator). The survey was referenced to the WGS84 geodetic system, and it used the EGM96 geoid model to represent the model orthometric heights. In areas with dense vegetation, the radars employed in the SRTM mission were unable to penetrate through the treetops, thereby it generated a DSM instead of a DEM. In general, the SRTM model has the technical specifications to achieve a vertical accuracy of 16 meters and a horizontal accuracy of 20 meters (Rodriguez et al., 2005).

The Advanced Spaceborne Thermal Emission and Reflection Radiometer Global Digital Elevation Model (ASTER GDEM), which is also used in this work, was conceived via a partnership between the Japan Ministry of Economy, Trade and Industry (METI) and NASA. The ASTER GDEM covers $99 \%$ of the Earth's continental land mass and it is delimited by latitudes from 83N to 83의 the DSM was created with stereoscopic images obtained from the ASTER sensor on board of the TERRA satellite between the years of 2000 and 2010. The generated DSM presents a spatial resolution of 1 arc second ( 30 meters at the equator), and it is also referenced to the WGS84 geodetic system and associated with the EGM96 geoid model. Likewise, version 2 of the GDEM (GDEM2) presents an absolute global planimetric accuracy of 6 meters and an absolute global altimetric accuracy of 12 meters (Tachikawa et al., 2011).

To evaluate the 3D positional accuracies of the SRTM-X and GDEM2 test DSM, another DSM provided by the Geoinformation and Technology Institute of the State of Minas Gerais (IGTEC) was used as reference. The reference DSM was obtained with digital aerial photogrammetry with a spatial resolution of 1.2 meters, being referenced to SIRGAS2000 geodetic system using the MAPGEO2010 model for the representation of orthometric heights. This DSM quality is compatible with $1 / 10,000$ scale and 5 meters vertical equidistance of contour lines. 


\subsection{Brazilian positional accuracy technical standard}

The Brazilian positional accuracy technical standard is defined by the Decree 89817 (1984), which defines the regulatory instructions for Brazilian cartography. This definition includes two tolerances known as: Cartographic Accuracy Standard (PEC - "Padrão de Exatidão Cartográfica" in Portuguese) and the Standard-Error (EP - "ErroPadrão" in Portuguese). These tolerances are used to analyze the horizontal and vertical accuracies separately, as they are dependent upon the map scale and contour interval.

According to BRASIL (1984), DSG (2011) and Santos (2016b), for a cartographic product to be classified according to Decree 89817, it must meet two conditions regarding the planimetry and the altimetry for the classification of the product, described below.

Conditions for planimetry: (i) $90 \%$ of the tested points must present planimetric discrepancies that are less than or equal to the value of the PEC concerning the map scale and the class being tested by and (ii) the root mean square (RMS) of the planimetric discrepancies must be less than or equal to the EP tolerance defined by the Standard (Table 1).

Conditions for altimetry: (i) $90 \%$ of the tested points must present altimetric discrepancies that are less than or equal to the value of the PEC regarding the contour interval and the class being tested, and (ii) the RMS of the altimetric discrepancies must be less than or equal to the EP tolerance.

Table 1: Planimetric and altimetric tolerances used in the Brazilian positional accuracy standard.

\begin{tabular}{ccccc}
\hline \multirow{2}{*}{ Class } & \multicolumn{2}{c}{ Planimetry } & \multicolumn{2}{c}{ Altimetry } \\
\cline { 2 - 5 } & PEC & EP & PEC & EP \\
\hline $\mathrm{A}$ & $0.5 \mathrm{~mm} * \mathrm{E}$ & $0.3 \mathrm{~mm} * \mathrm{E}$ & $0.5^{*} \mathrm{ci}$ & $0.33^{*} \mathrm{ci}$ \\
\hline $\mathrm{B}$ & $0.8 \mathrm{~mm} * \mathrm{E}$ & $0.5 \mathrm{~mm} * \mathrm{E}$ & $0.6^{*} \mathrm{ci}$ & $0.4^{*} \mathrm{ci}$ \\
\hline $\mathrm{C}$ & $1.0 \mathrm{~mm} * \mathrm{E}$ & $0.6 \mathrm{~mm} * \mathrm{E}$ & $0.75^{*} \mathrm{ci}$ & $0.5^{*} \mathrm{ci}$ \\
\hline & & $*$ Where, E: map scale denominator; ci: contour interval
\end{tabular}

Before classifying the positional accuracy according to the Brazilian technical standard, it is usual to perform the analysis of atypical data in the discrepancies sample. Consequently, the outliers in the data are detected, and then the two conditions described in the Brazilian technical standard are verified. If the conditions are met, the cartographic product is classified into one of three classes, namely, A, B or C, for analyzed scaled or vertical equidistance. If at least one of the two conditions fails, the product is not rated for tested class and scale.

\subsection{Methodology}

The methodology applied in this study was performed in three steps: (i) pre-processing the DSM; (ii) extracting the 3D homologous points from the DSM; and (iii) evaluating the positional planimetric and altimetric accuracies of the DSM according to the Decree 89817 (1984) classification.

In the pre-processing step of the DSMs, the first step was setting test and reference data to the same spatial reference systems. Both reference DSM and test DSM have different geoid models to represent the orthometric height. Therefore, in order to eliminate the influence of the elevation in the geoid models, the orthometric heights in the DSMs were converted to ellipsoidal heights according to Santos et al. (2016a) methodology. 
For the extraction of 3D homologous points from the DSMs, the methodology proposed in section 2 (Figures 2 and 3) was employed with ArcGIS 10.5 software (ESRI, 2014).

The workflow in ArcGIS consisted in eight steps: (i) fill the sinks in the model; (ii) obtain the flow direction using the D8 algorithm; (iii) calculate the cumulative flow; (iv) define a 10 hectares threshold to determine the beginning of the flow and to obtain the drainage network; $(v)$ obtain the confluence points (3D coordinates) of the numerical hydrography lines; (vi) obtain the accumulation basin from each confluence point; (vii) obtain the ridgelines from the delimited accumulation basins; (viii) obtain the ridgelines intersection points (3D coordinates).

After the extraction of ridge and confluence points from the test DSM (SRTM-X and GDEM2) and the reference DSM, 173 homologous points ( 95 ridge points and 78 confluence points), readily identifiable and distributed throughout the study area, were selected (Figure 8). For this selection, a systematic stratified sampling scheme was used partitioning the area into quadrants. In order to do so, quadrants with side of $1 \mathrm{~km}$ were created, and one or two points were acquired from each quadrant. Some quadrants, especially those along the boundaries of the study area, did not present well-defined homologous points, and thus, no checkpoints were selected from among them (see Figure 8).

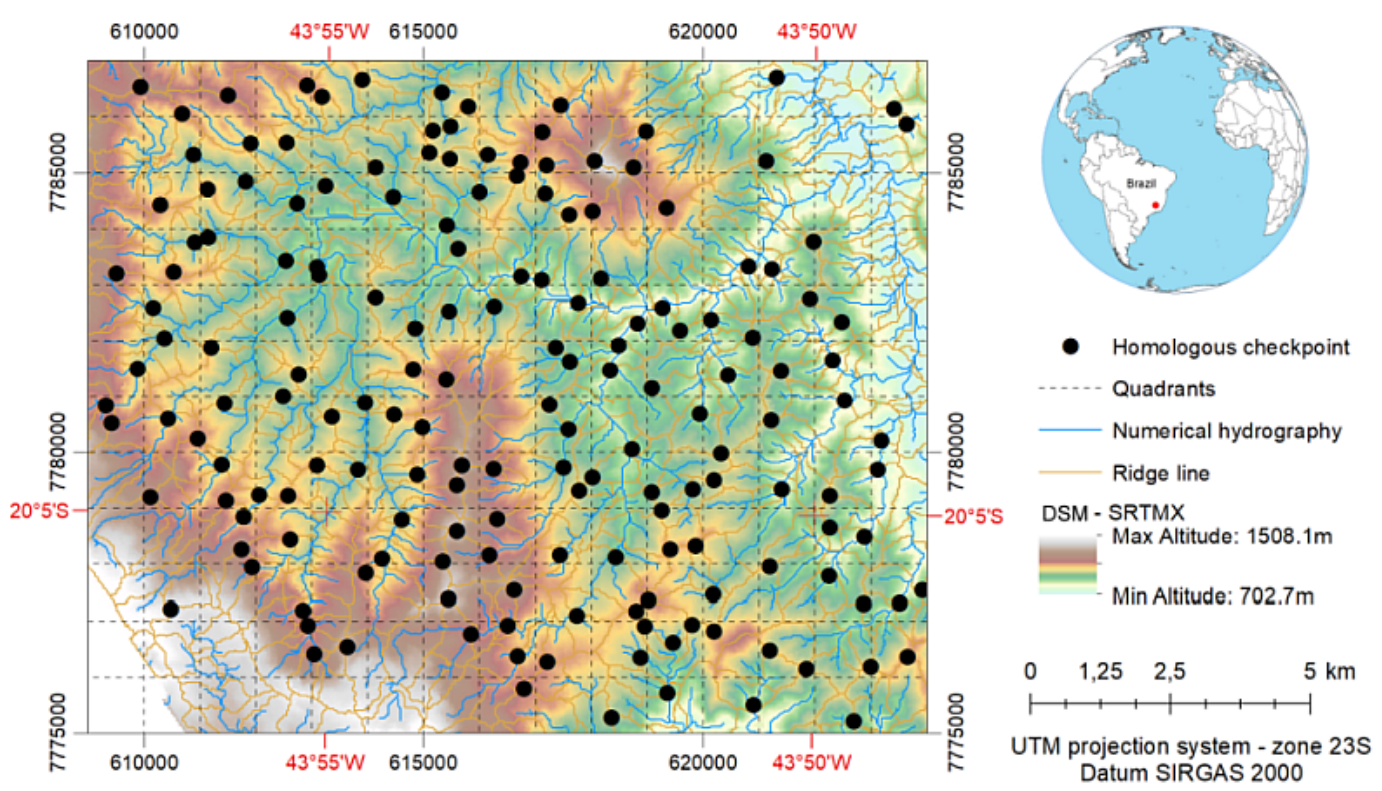

Figure 8: Distribution of 3D checkpoints in the study area.

To evaluate the planimetric and altimetric positional accuracies according to Decree 89817 (1984), the positional discrepancies were calculated based on the differences between the coordinates of the homologous points extracted from the test DSM (STRM-X and GDEM2) and those from the reference DSM. Afterwards, following the Brazilian technical standard, the planimetric and altimetric positional accuracies were evaluated separately according to section 3.2.

\section{Results and Discussion}

With the 173 checkpoints and their corresponding positional discrepancies, the outliers were detected using boxplot diagrams in R software (R Core Team, 2019). Figure 9 presents these boxplot diagrams for planimetric and altimetric discrepancies (in absolute values) for SRTM-X and GDEM2. A total of six outliers in SRTM-X and seven outliers in GDEM2 were detected. 
After that, an inspection in the checkpoints detected as outliers was performed. As no inconsistences were found in the process of extraction and identification of homologous points, all the studied points have been considered valid for the positional accuracy evaluation as discussed in Santos et al. (2016b), hence no data was discarded.

By analyzing Figure 9, it is possible to perceive higher dispersion of data in the planimetric component, whereas there is lower dispersion in the altimetric component. In general, SRTM-X and GDEM2 presented similar positional discrepancy magnitudes. Furthermore, Table 2 presents descriptive statistics of the planimetric and altimetric discrepancies sample. Figures 10 and 11 present charts of the checkpoints positional discrepancies from SRTM-X and GDEM2, respectively.

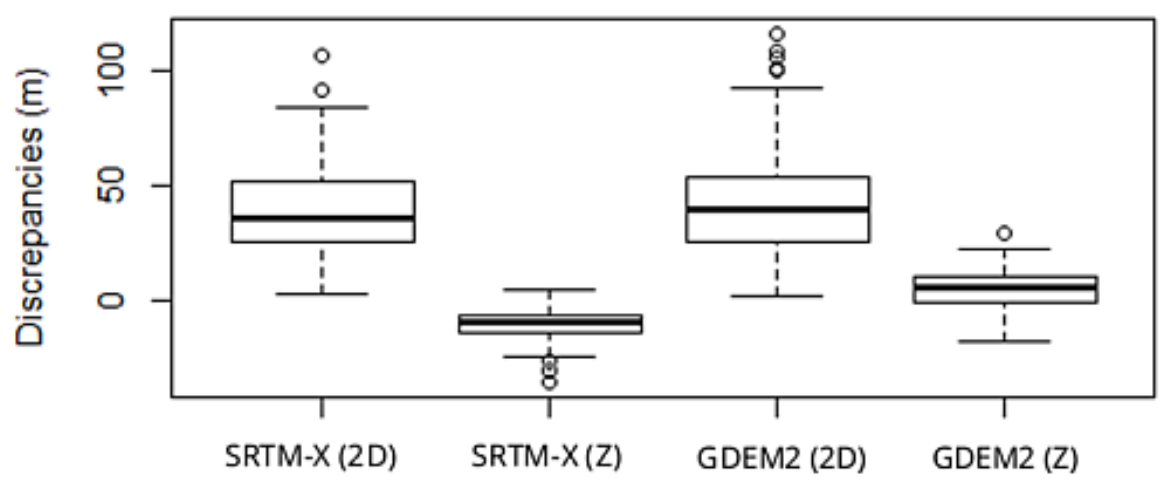

Figure 9: Detection of outliers through boxplot diagrams for the planimetric and altimetric discrepancies (absolute values) of SRTM-X and GDEM2.

Table 2: Descriptive statistics of the checking samples from SRTM-X and GDEM2 after the removal of outliers.

\begin{tabular}{ccccc}
\hline \multirow{2}{*}{ Statistics } & \multicolumn{2}{c}{ SRTM-X } & \multicolumn{3}{c}{ GDEM2 } \\
\cline { 2 - 5 } & $\mathrm{d} 2 \mathrm{D}$ & $\mathrm{dZ}$ & $\mathrm{d} 2 \mathrm{D}$ & $\mathrm{dZ}$ \\
\hline No. Points & 173 & -10.3 & 173 & 173 \\
\hline Average [m] & 39.7 & 12.3 & 42.0 & 4.9 \\
\hline RMS [m] & 44.8 & -35.4 & 17.8 & 9.6 \\
\hline Minimum [m] & 3.1 & 4.6 & 117.0 & -18.4 \\
\hline Maximum [m] & 106.9 & 29.6 \\
\hline
\end{tabular}

* Where, d2D: planimetric discrepancies; dZ: altimetric discrepancies

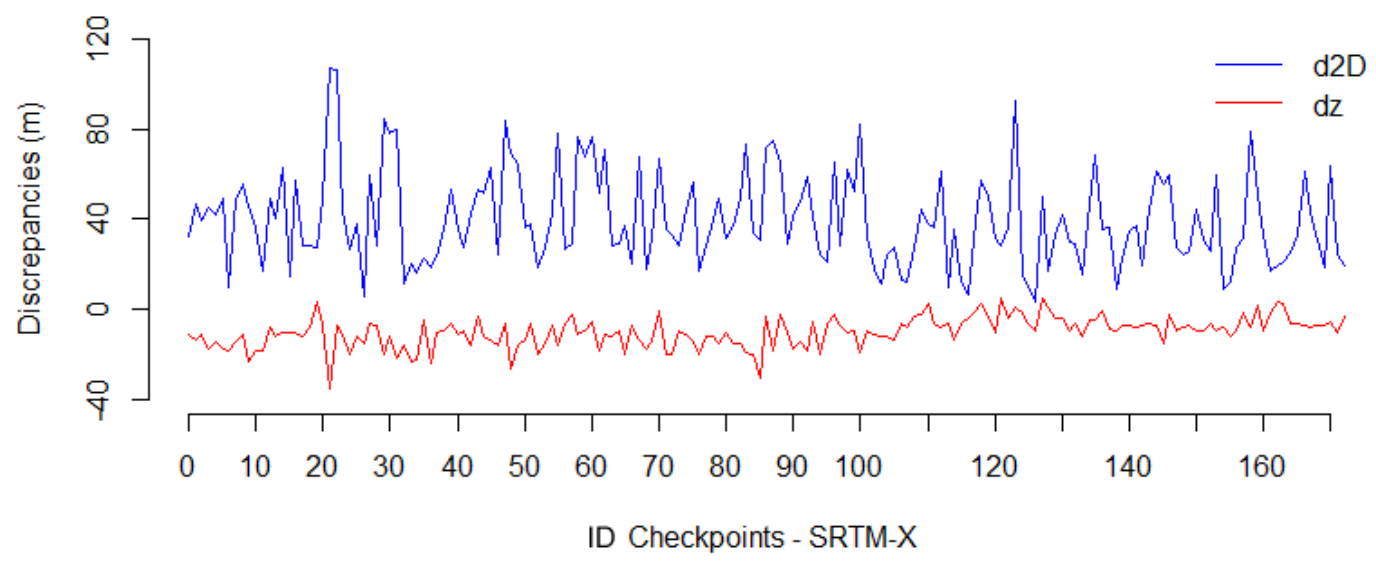

Figure 10: Chart of the positional discrepancies in the evaluation of the SRTM-X positional accuracy. 


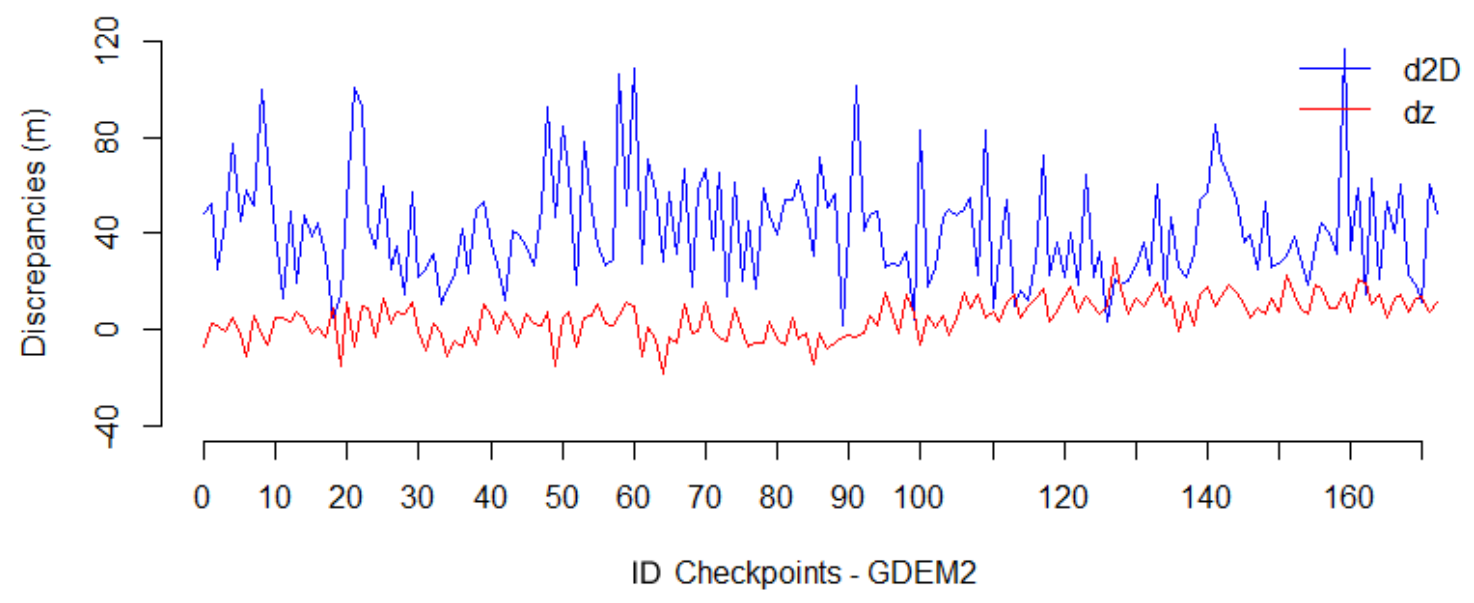

Figure 11: Chart of the positional discrepancies in the evaluation of GDEM2 positional accuracy.

It can be observed from the descriptive statistics (Table 2) that most of the altimetric discrepancies present negative values in SRTM-X, indicating that its altimetry is, on average, approximately $10 \mathrm{~m}$ lower than the reference DSM. Meanwhile, GDEM2 presented both positive and negative altimetric discrepancies, indicating that this DEM possibly has the same height level as the reference model, in general, however, it showed variations in some of the reference regions for the two models.

For the study area, the planimetric discrepancies are approximately four times greater than the altimetric discrepancies and from two to three times greater than the technical specifications of the evaluated DSMs.

When evaluating the planimetric and altimetric positional accuracies separately, as recommended by Decree 89817 (1984), both evaluated DSMs were classified into Classes A and B at 1:100,000 scale regarding the altimetry and planimetry, respectively. Tables 3 and 4 present the conditions and tolerances used for the classification of SRTM-X and GDEM2, respectively, according to the traditional methodology from Decree 89817 and using the method proposed in this work.

Table 3: Parameters and results of the evaluation of planimetric and altimetric accuracies of SRTM-X according to the traditional methodology of Decree 89817.

\begin{tabular}{ccccccc}
\hline Component & Scale (Class) & PEC & EP & $\%($ di $\leq$ PEC) & RMS(di) $\leq$ EP & Classification \\
\hline Altimetric & $1: 100,000(\mathrm{~A})$ & 25 & 16.7 & $98.3 \%$ & Attends & Approved \\
\hline \multirow{2}{*}{ Planimetric } & $1: 100,000(\mathrm{~A})$ & 50 & 30 & $72.8 \%$ & Failed & Disapproved \\
\cline { 2 - 7 } & $1: 100,000(\mathrm{~B})$ & 80 & 50 & $96.5 \%$ & Attends & Approved \\
\hline
\end{tabular}

*Where, di: discrepancies.

Table 4: Parameters and results of the evaluation of planimetric and altimetric accuracies of GDEM2 according to the traditional methodology of Decree 89817.

\begin{tabular}{ccccccc}
\hline Component & Scale (Class) & PEC & EP & $\%(\mathrm{di} \leq \mathrm{PEC})$ & $\mathrm{RMS}(\mathrm{di}) \leq \mathrm{EP}$ & Classification \\
\hline Altimetric & $1: 100,000(\mathrm{~A})$ & 25 & 16.7 & $100.00 \%$ & Attends & Approved \\
\hline \multirow{2}{*}{ Planimetric } & $1: 100,000(\mathrm{~A})$ & 50 & 30 & $66.4 \%$ & Failed & Disapproved \\
\cline { 2 - 7 } & $1: 100,000(\mathrm{~B})$ & 80 & 50 & $93.0 \%$ & Attends & Approved \\
\hline
\end{tabular}

*Where, di: discrepancies. 
Even though the literature (Giacomin et al., 2015; loannidis et al., 2014; Kolecka and Kozak, 2014; Rodriguez et al., 2005) either neglected or failed to explicitly state the planimetric uncertainties in the DSM during the verification of the vertical model accuracy and therefore used an erroneous model, as shown in Figure 1, the RMS of the altimetric discrepancies obtained in this work (12.3 $\mathrm{m}$ and $9.6 \mathrm{~m}$ for SRTM-X and GDEM2, respectively) are similar to most of the results mentioned above.

Tachikawa et al. (2011) evaluated the positional accuracy of GDEM2 in Japan and in the United States using over 18,000 checkpoints, obtaining a RMS for altimetric discrepancy of approximately $8.7 \mathrm{~m}$. For the planimetric discrepancy, the authors compared GDEM2 with several DSMs characterized by higher accuracies and obtained an uncertainty between 70 and 80 meters.

Moreover, Dong et al. (2015) evaluated the planimetric and altimetric accuracies of SRTM-X and GDEM2 using a more accurate reference DSM for a region of China. The average planimetric displacements for SRTM-X were $\mathrm{X}=-29.7 \mathrm{~m}$ and $y=43.7 \mathrm{~m}$, and the respective ones for GDEM2 were $x=23.3 \mathrm{~m}$ and $y=73.3 \mathrm{~m}$. The RMS altimetric discrepancies were $3.5 \mathrm{~m}$ and $6.5 \mathrm{~m}$ for SRTM-X and GDEM2, respectively.

Unlike the results obtained by Dong et al. (2015), the present work analyzed a region where GDEM2 showed a better altimetric RMS than the SRTM-X. Nevertheless, when it comes to planimetry, the work presented herein shows similar results to those from Dong et al. (2015) since the quality of SRTM-X was better than that of GDEM2. Furthermore, the altimetric accuracy obtained by Tachikawa et al. (2011) was similar to the results obtained in this work. Meanwhile, the average planimetric discrepancies were approximately 40 meters in this work while the cited authors obtained discrepancies ranging from 70 to 80 meters. These differences could be explained by the applied methods or mostly by the study region, which will change the area size, number of used points and topography.

\section{Conclusions}

Considering the experiments conducted herein, it is possible to see the potential and practical viability of the proposed methodology for the extraction of homologous points from a DEM/DSM to evaluate the positional accuracies of cartographic products. The methodology has shown itself to be easy to implement and efficient when it comes to reaching the objective. For future research, this methodology and the proposed evaluation of the 3D positional accuracy should be tested with different products and reliefs.

In general, the SRTM-X and GDEM2 presented similar classifications regarding the positional accuracy. Both DSMs presented Class B for planimetric accuracy and Class A for altimetric accuracy at 1:100,000 scale according to the traditional methodology of Decree 89817 used to evaluate the components separately.

Finally, the investigation of non-rigid methods for lines corresponding to the iterative closest point (ICP) is recommended to automate the correspondence process and the selection of homologous points extracted from test and reference DSMs.

\section{AUTHOR'S CONTRIBUTION}

Afonso Santos and Nilcilene Medeiros conceived of the presented idea. Afonso Santos developed the study, experiments and writing. All the authors contributed with preparation of draft manuscript, review of the proposed method, discussion of the results, data analysis, paper revision and refinement. 


\section{REFERENCES}

Ariza, F. J. L. and A. D. G. Atkinson. 2008. "Analysis of some positional accuracy assessment methodologies". Journal of Surveying Engineering, v. 134, $n^{\circ} 2$.

Barber, C. P., and A. Shortridge. 2005. "LiDAR elevation data for surface hydrologic modeling: Resolution and representation issues". Cartogr. Geogr. Inf. Sci., v. 32, n. 4, p. 401-410.

Boulton, S. J. and Stokes, M. 2018. "Which DEM is best for analyzing fluvial landscape development in mountainous terrains?". Geomorphology, v. 310.

BRASIL. 1984. Decree n. 89.817 of June 20, 1984. National Cartographic Technical Standard. (in portuguese). Brazil.

Dong, Y., H.C. Chang, W. Chen, K. Zhang and R. Feng. 2015. "Accuracy assessment of GDEM, SRTM, and DLR-SRTM in Northeastern China". Geocarto International.

DSG. 2011. ET-ADGV: Technical Specification for the Acquisition of Vector Geospatial Data. National Spatial Data Infrastructure (in portuguese). Brazil.

ESRI. 2014. ArcGIS.

Giacomin, G.; M. B. Carvalho, A. P. Santos, N. G. Medeiros and A. S. Ferraz. 2014. "Comparative analysis of interpolation methods for surface models". Brazilian Journal of Cartography (Revista Brasileira de Cartografia - in portuguese), $n^{\circ} 66 / 6$.

Goulden, T. C Hopkinson, R. Jamieson, and S Sterling. 2014. "Sensitivity of watershed attributes to spatial resolution and interpolation method of LiDAR DEMs in three distinct landscape". Water Resour. Res., 50, p. 1908-1927.

Gyasi-Agyei, Y., G. Willgoose, and F. P. Troch. 1995. "Effects of vertical resolution and map scale of digital elevation models on geomorphological parameters used in hydrology". Hydrol. Processes, v. 9, n.3-4, p. 363-382.

Höhle, J., and C. Pedersen. 2010. "A New Method for Checking the Planimetric Accuracy of Digital Elevation Models Data Derived by Airborne Laser Scanning." In Proceedings of the 9th International Symposium on Spatial Accuracy Assessment in Natural Resources and Environmental Sciences, University of Leicester, Leicester, UK, 253-56.

Ioannidis, C, E. Xinogalas and S. Soile. 2014. "Assessment of the global digital elevation models ASTER and SRTM in Greece". Survey Review, v. 46, n. 338, p. 342-354.

Li, J., and D. W. Wong. 2010. "Effects of DEM sources on hydrologic applications". Comput. Environ. Urban Syst., v. 34, n. 3, p. 251-261.

Li, Z., Q. Zhu and C. M. Gold. 2005. Digital Terrain Modelling. Principles and Methodology. New York.

Kolecka, N. and J. Kozak. 2014. "Assessment of the Accuracy of SRTM C- and X-Band High Mountain Elevation Data: a Case Study of the Polish Tatra Mountains". Pure Appl. Geophys, v. 171, p. 897-912.

Maas, H. 2002. "Methods for Measuring Height and Planimetry Discrepancies in Airborne Laserscanner Data." Photogrammetric Engineering \& Remote Sensing, v. 68, n. 9, p. 933-40.

Maune, D.F. 2007. Digital Elevation Model Technologies and Applications: the DEM User Manual, second ed. American Society for Photogrammetry and Remote Sensing, ASPRS, Bethesda, Maryland.

McMaster, K. J. 2002. "Effects of digital elevation model resolution on derived stream network positions. Water Resour. Res., v. 38, n.4.

Mozas, A. T. C., M. A. Ureña, and J. L. Pérez. 2013. "Accuracy of Contour Lines Using 3D Bands." International Journal of Geographical Information Science, v. 27, n. 12, p. 2362-74.

Murphy, P. N. C., J. Ogilvie, F. Meng, and P. Arp. 2008. "Stream network modelling using LiDAR and photogrammetric digital elevation models: A comparison and field verification”. Hydrol. Processes, v. 22, n.12, p. 1747-1754. 
O'Callaghan, J. F. and D. M. Mark. 1984. “The Extraction of Drainage Networks From Digital Elevation Data” Computer Vision, Graphics and Image Processing, 28: 328-344.

R Core Team. 2019. "R: A language and environment for statistical computing”. R Foundation for Statistical Computing. R Foundation for Statistical Computing, Vienna, Austria.

Reinoso, J. F. 2011. "An Algorithm for Automatically Computing the Horizontal Shift between Homologous Contours from DTMs." ISPRS Journal of Photogrammetry and Remote Sensing, v. 66, n. 3, p. 272-86.

Remmel, T. K., K. W. Todd, and J. Buttle. 2008. "A comparison of existing surficial hydrological data layers in a low relief forested Ontario landscape with those derived from a LiDAR DEM". For. Chron., v. 84, n.6, p. 850-865.

Rodriguez, E., C. S. Morris, J. Belz, E. Chapin, J. Martin, W. Daffer, and S. Hensley. 2005. "An Assessment of the SRTM Topographic Products". Technical Report JPL D-31639, Jet Propulsion Laboratory, Pasadena, California, United States of America.

Santos, A. P., N. G. Medeiros, D. D. Rodrigues and G. R. Santos. 2016a. "Planimetric positional accuracy assessment in digital surface models with the use of linear features". Bulletin of Geodetic Sciences (Boletim de Ciências Geodésicas in portuguese), v. 22, nº 1, p.157-174.

Santos, A. P., D. D. Rodrigues, N. T. Santos and J. GRIPP Jr. 2016b. "Assessment of positional accuracy in spatial data using techniques of spatial statistics: proposal of a method and an example using the Brazilian standard". Bulletin of Geodetic Sciences (Boletim de Ciências Geodésicas - in portuguese), v. 22, n4, p.630-650.

Tachikawa, T., M. Hato, M. Kaku, and A. Iwasaki. 2011. "The characteristics of ASTER GDEM version 2". IGARSS.

Tarboton, D. G. 1997. "A New Method for the Determination of Flow Directions and Contributing Areas in Grid Digital Elevation Models," Water Resources Research, 33(2): 309-319

Vosselman, G. 2008. "Analysis of Planimetric Accuracy of Airborne Laser Scanning Surveys." In International Archives of Photogrammetry and Remote Sensing, XXXVII:99-104.

Wilson, J. P. 2018. Title: Environmental applications of digital terrain modeling. Hoboken, NJ: Wiley-Blackwell. 